\title{
Preclinical evaluation and reverse phase protein Array-based profiling of PI3K and MEK inhibitors in endometrial carcinoma in vitro
}

\author{
Ozlem Aslan ${ }^{1 *}$, Mattia Cremona ${ }^{1}$, Clare Morgan ${ }^{1}$ Lydia W. Cheung ${ }^{2}$, Gordon B. Mills $^{2}$ and Bryan T. Hennessy ${ }^{1}$
}

\begin{abstract}
Background: The phosphoinositide-3-kinase (PI3K) pathway is the most commonly activated pathway in cancers due to mutations at multiple nodes and loss of PTEN. Furthermore, in endometrial cancer (EC), PI3K and RAS/RAF/ MEK/MAPK (RAS/MAPK herein) pathway mutations frequently co-exist. We examined the role of PI3K and RAS/MAPK pathway mutations in determining responsiveness to therapies targeted to these pathways in vitro in EC.

Methods: 13 EC cell lines were profiled for their PI3K pathway and KRAS mutational and PTEN protein status and treated with one MEK- and two PI3K- targeted inhibitors alone and in combination. Expression and phosphorylation of 66 proteins were evaluated by Reverse-Phase-Protein-Array (RPPA) in 6 EC cell lines to identify signalling changes in these pathways in response to therapy.

Results: PTEN protein loss and the absence of any tested pathway mutations are dominant negative predictors of sensitivity to MEK inhibition. KRAS-mutated cells were most sensitive to MEK inhibition, but significantly more resistant to PI3K inhibition than KRAS-wild-type cell lines. Combinations of PI3K and MEK inhibitors showed synergy or additivity in all but two cell lines tested. Treatment of KRAS-mutated cells with PI3K inhibitors and treatment of PTEN-low cells with a MEK inhibitor were most likely to induce activation of MEK/MAPK and AKT, respectively, likely indicative of feedback-loop regulation.

Conclusions: MEK inhibition may be a promising treatment modality, not just for ECS with mutated KRAS, but also for those with retained PTEN. Up-regulation of MEK/MAPK signalling by PI3K inhibition, and up-regulation of AKT activation by MEK inhibition may serve as potential biomarkers of likely responsiveness to each inhibitor.
\end{abstract}

Keywords: Endometrial cancer, Biomarkers, PTEN loss, PIK3CA, KRAS, PI3K inhibitor, MEK inhibitor, Protein signalling

\section{Background}

The phosphoinositide 3-kinase (PI3K) pathway is commonly activated in many cancer types, including EC, frequently due to mutations at multiple nodes, for example PIK3CA (which encodes the p110 $\alpha$ catalytic subunit of PI3K), or loss of PTEN protein expression (the major tumour suppressor that regulates this pathway). The RAS/MAPK pathway has also been shown to be a good target for novel therapy in preclinical cancer models. Additionally, bidirectional crosstalk with

\footnotetext{
* Correspondence: ozlemasl@gmail.com

${ }^{1}$ Department of Medical Oncology, Royal College of Surgeons in Ireland, Beaumont Hospital, Dublin 9, Ireland

Full list of author information is available at the end of the article
}

the PI3K pathway $[1,2]$ suggests that interactions between these two pathways might dictate the responsiveness of cancer cells to inhibitors targeted against these pathways [3]. We have previously performed an integrated analysis of the PI3K and interacting pathways in 243 endometrial cancer and showed that the PI3K pathway is activated in over $80 \%$ of endometrioid endometrial cancers by mutations in the PTEN (44\%), PIK3CA (40\%), PIK3R1 (20\%) and PIK3R2 (5\%) genes $[4,5]$. In terms of the RAS/MAPK pathway, we found activating KRAS mutations in approximately $20 \%$ of endometrial cancers $[6,7]$. The coexistence of mutations in KRAS and PI3K pathway members suggests that the RAS/ MAPK and PI3K pathways may interact as critical drivers of 
pathogenesis in EC and serve as targets for the development of novel cancer treatments. However, the implications of these mutations for EC responsiveness to therapies targeted to these pathways have not been fully defined.

Novel inhibitors targeting the PI3K pathway and RAS/ MAPK pathway (e.g. MEK inhibitors) are currently being developed. Pictilisib (GDC-0941) and apitolisib (GDC0980) are potent inhibitors of class I PI3K isoforms [8], class I PI3K and mTOR-kinase (TORC1/2), respectively [9, 10], and cobimetinib (GDC-0973) is a selective smallmolecule inhibitor of MEK that is highly potent [11]. Targeting both pathways has been shown to be more effective in preclinical cancer models than targeting either pathway alone [11-13]. Here, we investigated the effects of mutations in the PI3K and RAS/MAPK pathways on tumour cell signalling and on responsiveness to these PI3K and RAS/ MAPK-targeted therapies alone and in combination in EC.

\section{Methods}

\section{EC cell lines and cell culture}

The human endometrial cancer cell lines AN3CA, HEC1A, HEC1B, HEC50, ECC1, EFE184, ETN1, KLE and SKUT2 were obtained from the Department of Systems Biology, the University of Texas M.D. Anderson Cancer Center (Houston, USA), EN, MFE296 and MFE280 cell lines were purchased from the German Collection of Microorganisms and Cell Cultures (DSMZ; Braunschweig, Germany). EN-1078D cell line was kindly provided by Prof Eric Asselin (University of Quebec), where informed consent was attained and research studies approved by the Montreal University Institutional Review Board [14]. The EC cell lines AN3CA, HEC1A, KLE and SKUT2 cell lines were grown in DMEM-F12 (Sigma \#D6421), with 10\% FBS (Lonza \#DE14-801F); EN-1078D was grown in DMEM-F12 (Sigma \#D6421) and 10\% FBS with the addition of $50 \mu \mathrm{g} / \mathrm{mL}$ gentamicin (Invitrogen \#15710049), HEC50 was grown in DMEM-F12 with the addition of $2 \mathrm{mM}$ glutamine (Sigma \#D8437) and 10\% FBS. ECC1, EFE184, EN, ETN1 and MFE280 were grown in RPMI1640 (Sigma \#R8758) supplemented with 10\% FBS. MFE296 was grown in RPMI-1640 (Sigma \#R8758) with $10 \% \mathrm{FBS}$ and insulin-transferrin-selenium-X (Invitrogen \#51500). HEC1B and HEC59 were grown in MEM (Sigma \# 2279) with the addition of $10 \%$ FBS, $1 \%$ non-essential amino acids and $10 \mathrm{~mL}$ glutamine (Sigma \#D8437). Growth media were supplemented with $100 \mu \mathrm{g} / \mathrm{ml}$ streptomycin and $100 \mathrm{U} / \mathrm{ml}$ penicillin (Invitrogen) and all cell lines were cultured at $37{ }^{\circ} \mathrm{C}$ in $5 \% \mathrm{CO}_{2}$. Cell lines were fingerprinted at the beginning of the study to confirm their identities.

\section{Cell line somatic mutation genotyping}

Genomic DNA was extracted using QIAamp DNA Mini Kit (Qiagen). We designed high-throughput assays for somatic mutations in KRAS, PIK3CA, PIK3R1, PIK3R2 and
PTEN, and applied a mass spectroscopy-based approach to detect single nucleotide polymorphisms (MassARRAY, Sequenom) as described previously $[5,15]$.

\section{Cell viability assays}

The inhibitors pictilisib (GDC-0941), apitolisib (GDC0980) and cobimetinib (GDC-0973) were obtained from Genentech, Inc. $1 \times 10^{4}$ cells/well were plated into flatbottomed, 96-well plates and allowed to attach overnight. All drug treatments including combinations were tested in triplicate during a 5-day incubation period with serial dilutions of drug in a final volume of $200 \mathrm{uL}$. Drug-free controls were included in each assay. DMSO controls were also performed for each assay. Plates were incubated at $37^{\circ}$ $\mathrm{C}$ in a humidified atmosphere with $5 \% \mathrm{CO}_{2}$ and cell viability was determined using an acid phosphatase assay as described previously $[16,17]$.

Protein extraction and reverse phase protein Array (RPPA) RPPA is a high through-put antibody based technique used for functional proteomic assessments of a large number of tumour samples and it offers a platform for comparison of the relative protein expression between these samples [18]. We plated cells in 6-well plates, allowed them to reach $60-80 \%$ confluence and extracted their proteins as described by us and others previously $[15,19,20]$. Cells were washed with cold PBS and lysed with ice-cold lysis buffer (1\% Triton X-100, $50 \mathrm{~mm}$ HEPES, pH 7.4, $150 \mathrm{mM} \mathrm{NaCl}, 1.5 \mathrm{mM} \mathrm{MgCl} 2,1 \mathrm{mM}$ EGTA, $100 \mathrm{mM} \mathrm{NaF}, 10 \mathrm{mM}$ Na pyrophosphate, $1 \mathrm{mM}$ Na3VO4, 10\% glycerol) supplemented with proteinase inhibitors (Roche Applied Science, Indianapolis, IN) as described previously $[19,21]$.

For RPPA, four-fold serial dilutions of protein extracts from EC cell lines were performed. Serially diluted lysates were arrayed on Oncyte Avid nitrocellulose-coated slides (Grace Bio-Labs, Bend, OR) using a QArray 2 arrayer (Molecular Devices, Wokingham, UK). Diluted samples were robotically printed on multiple slides that included positive and negative controls. Slides were probed with primary antibodies (see Additional file 1: Table S1) followed by a secondary antibody - either goat anti-rabbit IgG (1:5000) (Vector Laboratories, Burlingame, CA) or rabbit anti-mouse IgG (1:10) (Dako) depending on the particular primary antibody. Signals were amplified using a Dakocytomationcatalysed system (Dako) and visualized by chromogenic detection (using diaminobenzidine). Slides were then scanned, analysed, and quantified using customized software (Microvigene, VigeneTech Inc.) to generate spot intensities.

Heat-maps were generated from normalized data using unsupervised hierarchical clustering analysis performed with publicly available Morpheus software (https://software.broadinstitute.org/morpheus/). 


\section{Statistical analysis}

Drug combination assays synergy was assessed using the combination index method [22] using CompuSyn software (Combosyn Inc., Paramus, NJ, USA) [12]. A combination index $(\mathrm{CI})$ of $<1$ is considered to indicate synergy, $=1$ is considered additive and $>1$ is considered antagonistic [22]. We applied the Student's t-test to test the significance of pair-wise comparisons using GraphPad-Prism.5. P-value < 0.05 was considered statistically significant.

\section{Results}

\section{Genomic and proteomic features of the endometrial cancer cell lines}

By applying a mass spectroscopy-based analysis (MassARRAY, Sequenom) to the $13 \mathrm{EC}$ cell lines we confirmed their mutational status. As we and others have previously shown in primary endometrioid endometrial carcinomas (EECs) $[23,24]$, we found that multiple members of the PI3K pathway and sometimes KRAS were mutated in the same cell lines, including concomitant mutations (Tables 1 and 2).

Further, using RPPA, we found that PTEN protein expression was retained in the majority of the $\mathrm{EC}$ cell lines with the exception of three cell lines (EN, AN3CA, HEC59) which are all PTEN-mutated and had relatively high levels of phosphorylated AKT. We observed that the presence of a PTEN mutation was significantly associated with activation of PI3K pathway as determined by high levels of AKT phosphorylation at $\mathrm{Thr}^{308}(P=0.005)$ and at $\operatorname{Ser}^{473}(P=0.008)$ in EC cell lines, however no significant association was observed between PIK3CA, PIK3R1 or
KRAS mutation and AKT phosphorylation levels (see Additional file 2: Table S2). These results are inconsistent with previous studies in breast cancer cell lines [15] and endometrial cancer cell lines [24], providing PTEN mutation or PTEN loss as important activators of PI3Kmediated pro-survival signalling through AKT.

Unsupervised hierarchical clustering revealed three major cell line clusters (designated as C1, C2 and C3) (Fig. 1). Cluster $\mathrm{C} 1$ contained one cell line only, the PTEN-mutated MFE296, distinguished by high levels of PTEN, total MAPK-ERK1/2 expression and MAPKERK1/2 phosphorylation at $\mathrm{Thr}^{202} / \mathrm{Tyr}^{204}$. Cluster C2 and C3 were composed of four (HEC1A, MFE280, EFE184, KLE) and five (AN3CA, EN-1078D, HEC1B, HEC50, SKUT2) cell lines, respectively (Fig. 1). Cell line cluster $\mathrm{C} 2$ was composed of three of the PTEN-mutated cell lines (EN, HEC59, ETN1) of which two expressed low levels of PTEN (EN, HEC59). As compared with cluster C3, cell lines in cluster C2 contained higher levels of higher levels of phosphorylated AKT at Ser ${ }^{473}$ and $\mathrm{Thr}^{308}$, phosphorylated c-Raf at $\mathrm{Ser}^{338}$, phosphorylated S6 ribosomal protein at $\mathrm{Ser}^{235 / 236}$ and P70 S6 kinase, and lower levels of phosphorylated NFk $\beta$ p $65^{\operatorname{Ser} 536}$, phosphorylated MEK1/2 $2^{\text {Ser217/221, }}$ phosphorylated MAPK-ERK $1 / 2^{\text {Thr202/Tyr204 }}$ and phosphorylated GSK$3 \beta^{\text {Ser9 }}$. Thus it seems that cluster $\mathrm{C} 2$ is defined by activation of the PI3K pathway and cluster C3 by activation of the RAS/MAPK pathway. Unsupervised hierarchical clustering of the 66 proteins also revealed eight protein clusters (designated P1 to P8) (Fig. 1).

Table $1 I_{50}$ s of endometrial cancer cell lines $(n=13)$ to the PI3Ki pictilisib, PI3K/mTORi apitolisib and the MEKi cobimetinib as single agents $(n=13)$, and the combination of pictilisib with cobimetinib and apitolisib with cobimetinib $(n=11)$

\begin{tabular}{|c|c|c|c|c|c|c|c|c|c|}
\hline \multirow[t]{2}{*}{ Cell Line } & \multirow[t]{2}{*}{ Mutational status } & \multirow[t]{2}{*}{$\begin{array}{l}\text { PTEN } \\
\text { expression }\end{array}$} & \multirow{2}{*}{$\begin{array}{l}\text { PI3Ki Pictilisib (uM) } \\
I_{50}\end{array}$} & \multirow{2}{*}{$\begin{array}{l}\text { PI3K/mTORi } \\
\text { Apitolisib (uM) } \\
\mathrm{IC}_{50}\end{array}$} & \multirow{2}{*}{$\begin{array}{l}\text { MEKi } \\
\text { Cobimetinib (UM) } \\
\mathrm{IC}_{50}\end{array}$} & \multicolumn{2}{|c|}{$\begin{array}{l}\text { Combination of } \\
\text { Pictilisib:Cobimetinib } \\
\text { (uM) }\end{array}$} & \multicolumn{2}{|c|}{$\begin{array}{l}\text { Combination of } \\
\text { Apitolisib:Cobimetinib } \\
\text { (uM) }\end{array}$} \\
\hline & & & & & & $\mathrm{IC}_{50}$ & $\mathrm{Cl}$ at $\mathrm{ED}_{75}$ & IC50 & $\mathrm{Cl}$ at $\mathrm{ED}_{75}$ \\
\hline SKUT2 & PIK3CA only & High & 0.413 & 0.196 & 0.101 & 0.096 & 0.138 & 0.049 & 0.152 \\
\hline MFE280 & PIK3CA only & High & 0.440 & 0.247 & 0.461 & 0.207 & 0.357 & 0.091 & 0.528 \\
\hline EN1078D & PTEN + PIK3R1 & High & 0.242 & 0.079 & 0.178 & 0.103 & 0.634 & 0.057 & 0.468 \\
\hline MFE296 & PTEN + PIK3CA & High & 0.273 & 0.106 & 0.301 & 0.383 & 1.037 & 0.120 & 0.650 \\
\hline ETN1 & PTEN & High & 0.544 & 0.387 & 1.077 & 0.174 & 0.426 & 0.177 & 0.495 \\
\hline AN3CA & PTEN + PIK3R1 & Low & 0.057 & 0.028 & 0.518 & 0.339 & 0.129 & 0.070 & 0.142 \\
\hline HEC59 & PTEN + PIK3R1 & Low & 0.189 & 0.018 & 3.289 & - & - & - & - \\
\hline EN & $P T E N+P I K 3 C A+P I K 3 R 1$ & Low & 1.597 & 0.548 & 36.511 & 3.117 & 1.555 & 2.175 & 1.200 \\
\hline HEC1A & $K R A S+P I K 3 C A$ & High & 2.054 & 0.214 & 0.012 & - & - & - & - \\
\hline HEC1B & $K R A S+P I K 3 C A$ & High & 0.798 & 0.704 & 0.033 & 0.050 & 0.031 & 0.054 & 0.035 \\
\hline HEC50 & $K R A S+P I K 3 R 1$ & High & 0.247 & 0.210 & 0.001 & 0.131 & 4.921 & 0.169 & 6.613 \\
\hline EFE184 & WT for all & High & 0.372 & 0.368 & 0.149 & 0.074 & 0.433 & 0.157 & 0.328 \\
\hline KLE & WT for all & High & 2.796 & 7.253 & 39.337 & 0.644 & 0.142 & 0.144 & 0.005 \\
\hline
\end{tabular}

Drug combinations exhibiting antagonistic effects $(\mathrm{Cl}$ values $>1)$ are in shown bold

$I C_{50}$ inhibitor concentration to inhibit $50 \%$ of the cell growth, PI3Ki PI3K inhibitor, $\mathrm{PI} 3 \mathrm{~K} / \mathrm{mTORi} \mathrm{PI3K} / \mathrm{mTOR}$ inhibitor, $M E K i \mathrm{MEK}$ inhibitor, $C I$ Combination Index, $E D_{75}$ Effective Dose exhibiting $75 \%$ of loss in cell viability 
Table 2 Classification of endometrial cancer cell lines into four groups

\begin{tabular}{|c|c|c|c|c|c|c|c|}
\hline Groups & Cell line & PIK3CA & KRAS & PTEN & PIK3R1 & PIK3R2 & $\begin{array}{l}\text { PTEN } \\
\text { expression }\end{array}$ \\
\hline \multirow{2}{*}{$\begin{array}{l}\text { Group } 1 \text { (PIK3CA- } \\
\text { mutated only) }\end{array}$} & SKUT2 & E545K (hetero) & Wild-type & Wild-type & Wild-type & Wild-type & High \\
\hline & MFE280 & H1047Y (hetero) & Wild-type & Wild-type & Wild-type & Wild-type & High \\
\hline \multirow{3}{*}{$\begin{array}{l}\text { Group 2a (PTEN-mutated, } \\
\text { PTEN-retained) }\end{array}$} & EN1078D & Wild-type & Wild-type & Y88C & G376R (hetero) & Wild-type & High \\
\hline & MFE296 & $\begin{array}{l}\text { P539R (hetero); } \\
\text { I20M (hetero) }\end{array}$ & Wild-type & $\begin{array}{l}\text { R130Q (homo); N322 fs } \\
\text { (hetero) }\end{array}$ & Wild-type & Wild-type & High \\
\hline & ETN1 & Wild-type & Wild-type & $\begin{array}{l}\text { I122V (hetero), R130L } \\
\text { (hetero); N328 fs (hetero) }\end{array}$ & Wild-type & Wild-type & High \\
\hline \multirow{3}{*}{$\begin{array}{l}\text { Group } 2 b \text { (PTEN-mutated } \\
\text { and PTEN-Loss) }\end{array}$} & AN3CA & Wild-type & Wild-type & R130fs (homo) & REID557del (hetero) & Wild-type & Low \\
\hline & HEC59 & Wild-type & Wild-type & $\begin{array}{l}\text { Y46H (hetero); R233 } \\
\text { (hetero); P246L (hetero) }\end{array}$ & $\begin{array}{l}\text { T473S (hetero); K567E } \\
\text { (hetero); S460 fs (hetero) }\end{array}$ & Wild-type & Low \\
\hline & EN & T1025A (hetero) & Wild-type & K266 fs (hetero) & N260S (hetero) & Wild-type & Low \\
\hline \multirow[t]{3}{*}{ Group 3 (KRAS-mutated) } & HEC1A & G1049R (hetero) & $\begin{array}{l}\text { G12D } \\
\text { (homo) }\end{array}$ & Wild-type & Wild-type & Wild-type & High \\
\hline & HEC1B & G1049R (hetero) & $\begin{array}{l}\text { G12D } \\
\text { (homo) }\end{array}$ & Wild-type & Wild-type & Wild-type & High \\
\hline & HEC50 & Wild-type & $\begin{array}{l}\text { G12D } \\
\text { (hetero) }\end{array}$ & Wild-type & E468InsGEYDRLYE (homo) & Wild-type & High \\
\hline \multirow[t]{2}{*}{ Group 4 (Wild-type) } & EFE184 & Wild-type & Wild-type & Wild-type & Wild-type & Wild-type & High \\
\hline & KLE & Wild-type & Wild-type & Wild-type & Wild-type & Wild-type & High \\
\hline
\end{tabular}

The 6 cell lines highlighted in bold were selected for further RPPA analyses

\section{Sensitivity of endometrial cancer cell lines to two PI3K inhibitors and a MEK inhibitor}

We observed that EC cell lines possessing a KRAS mutation (HEC50, HEC1A and HEC1B) were more sensitive to the MEK inhibitor cobimetinib $\left(\mathrm{IC}_{50} \mathrm{~S}\right.$ from 0.001 to $0.033 \mu \mathrm{M})$ when compared with the other cell lines tested ( $\mathrm{IC}_{50}$ s from 0.101 to $39.337 \mu \mathrm{M}$ ) (Table 1 ). Conversely, EC cell lines possessing a PTEN mutation (MFE296, EN1078D, ETN1, AN3CA, HEC59, EN) were more resistant to MEK inhibition ( $\mathrm{IC}_{50} \mathrm{~s}$ from 0.178 to $36.511 \mu \mathrm{M}$ ) when compared with the cell lines without a PTEN mutation ( $\mathrm{IC}_{50} \mathrm{~s}$ from 0.101 to $0.001 \mu \mathrm{M}$ (with the exception of the wild-type cell line $\mathrm{KLE}$ with a $\mathrm{IC}_{50}$ of $39.337 \mu \mathrm{M}$ ).

In terms of the dual inhibition of PI3K and mTOR, we observed that EC cell lines with coexisting PTEN and PIK3R1 mutations (HEC59, AN3CA and EN-1078D) were more sensitive to the $\mathrm{PI} 3 \mathrm{~K} / \mathrm{mTOR}$ inhibitor apitolisib ( $\mathrm{IC}_{50}$ s from 0.018 to $0.079 \mu \mathrm{M}$ ) when compared with the others $\left(\mathrm{IC}_{50} \mathrm{~s}\right.$ from 0.196 to $7.253 \mu \mathrm{M}$ ) (Table 1 ). Two wild-type cell lines (EFE184, KLE) showed different responses to all three inhibitors, whilst KLE was very resistant to pictilisib, apitolisib and cobimetinib treatments $\left(\mathrm{IC}_{50} \mathrm{~S} 2.796,7.253\right.$ and $39.337 \mu \mathrm{M}$, respectively), the other wild-type cell line EFE184 was markedly sensitive with the $\mathrm{IC}_{50} \mathrm{~s}$ of $0.372,0.368$ and $0.149 \mu \mathrm{M}$ for pictilisib, apitolisib and cobimetinib, respectively (Table 1).

Next, we examined if expression levels of PTEN protein play an important role in response to therapies targeted to the PI3K and MEK pathways. Thus, we compared EC cell lines with PTEN protein loss (AN3CA, EN and HEC59) against EC cell lines with retained PTEN protein, and found that cell lines exhibiting PTEN loss were more sensitive to the PI3K inhibitor pictilisib $\left(\mathrm{IC}_{50} \mathrm{~S}\right.$ from 0.057 to $1.597 \mu \mathrm{M})$ when compared with cell lines with retained PTEN expression ( $\mathrm{IC}_{50} \mathrm{~S}$ from 0.242 to $\left.2.796 \mu \mathrm{M}\right)$. In terms of apitolisib sensitivity, cell lines with PTEN loss were more sensitive $\left(\mathrm{IC}_{50} \mathrm{~s}\right.$ from 0.018 to $\left.0.548 \mu \mathrm{M}\right)$ when compared with PTEN retained cell lines $\left(\mathrm{IC}_{50} \mathrm{~s}\right.$ from 0.106 to $7.253 \mu \mathrm{M}$ ). However, these cell lines with PTEN loss were more resistant to MEK inhibition $\left(\mathrm{IC}_{50} \mathrm{~s}\right.$ from 0.518 to $36.511 \mu \mathrm{M})$ when compared with cell lines with retained PTEN expression $\left(\mathrm{IC}_{50} \mathrm{~s}\right.$ from 0.001 to $1.077 \mu \mathrm{M}$ ), (again with the exception of the MEK inhibitor resistant wild-type KLE cell line with high levels of PTEN), indicating PTEN loss as a dominant marker of sensitivity to PI3K inhibition and resistance to MEK inhibition (Fig. 2c).

\section{Sensitivity of EC cell lines to the two PI3K inhibitors and a MEK inhibitor in combination}

Next, we sought to examine whether dual inhibition of both the PI3K and MEK pathways might result in synergistic effects on cell viability. We found that the combination of pictilisib with cobimetinib, and the combination of apitolisib with cobimetinib both resulted in synergistic growth inhibition in most of the cell lines tested (2/10), with the exception of 2 cell lines (EN, HEC50) (Table 1, Fig. 4a-b). Endometrial cancer cell lines in which synergism was observed to the combination of pictilisib (PI3Ki), apitolisib 


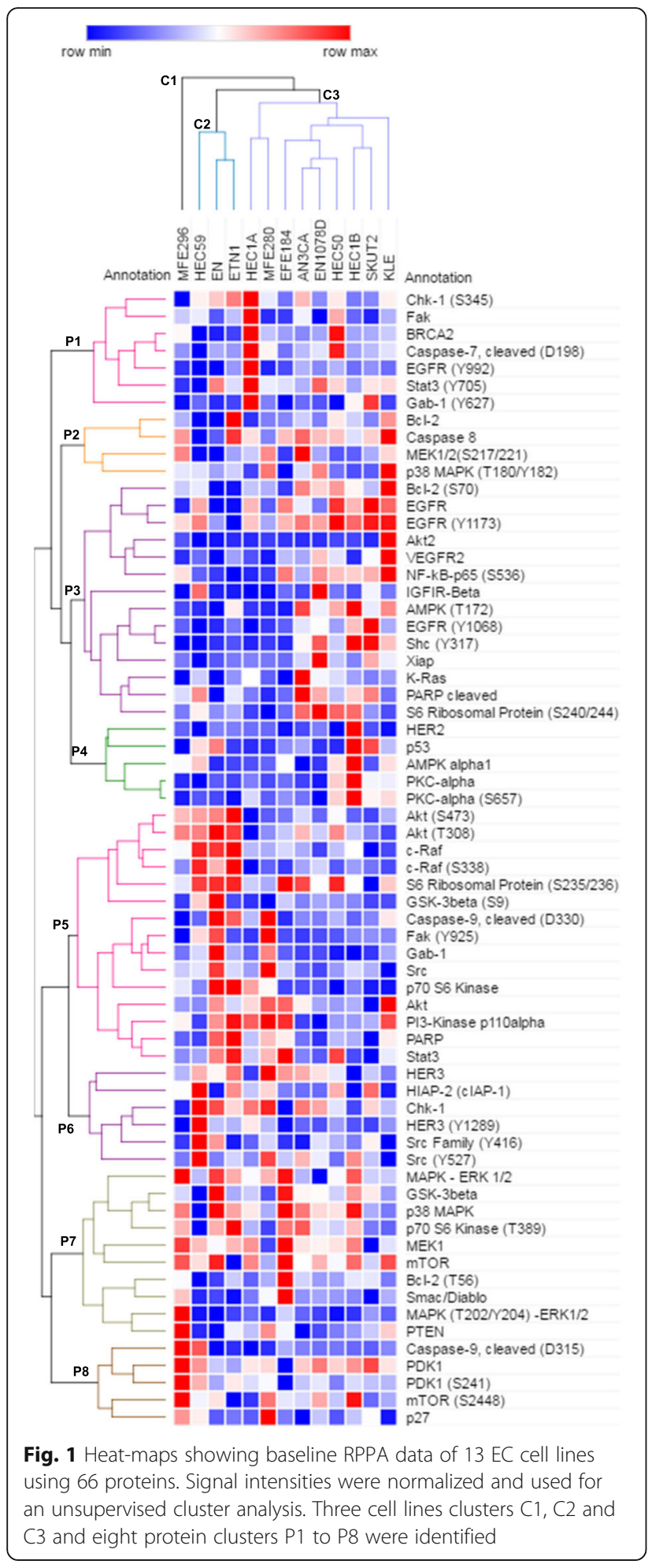

(PI3K/mTORi) and cobimetinib (MEKi) are shown in Additional file 3: Figure S1.

We observed strong antagonism as indicated by $\mathrm{CI}$ values greater than 1 (see Materials and Methods), for EN cell line with the combination of pictilisib and cobimetinib
$(\mathrm{CI}$ value $=1.555)$, and with the combination of apitolisib and cobimetinib (CI value $=1.200$ ). This antagonism may occur due to the high resistance to the MEK inhibitor cobimetinib in this cell line $\left(\mathrm{IC}_{50}: 36.511 \mu \mathrm{M}\right)$ when compared with pictilisib and apitolisib therapies alone $\left(\mathrm{IC}_{50} \mathrm{~S}\right.$ 1.597 and $0.548 \mu \mathrm{M}$, respectively) (Table 1 ). We also determined strong antagonism for HEC50 cell line with the combination of pictilisib and cobimetinib, and with the combination of apitolisib and cobimetinib (CI values 4.921 and 6.613, respectively). In contrast to EN cell line, antagonism in HEC50 cell line may occur due to the high sensitivity to MEK inhibition $\left(\mathrm{IC}_{50} 0.001 \mu \mathrm{M}\right)$ (Table 1 ).

HEC50 and EN cell lines in which antagonism was observed to the combination of pictilisib (PI3Ki), apitolisib (PI3K/mTORi) and cobimetinib (MEKi) are shown in Fig. 3.

\section{Classification of endometrial cancer cell lines}

We detected differential responsiveness to the PI3K inhibitors and to the MEK inhibitor between the cell lines with the presence of PTEN mutations and PTEN loss being significantly associated with PI3K pathway activation, enhanced sensitivity to PI3K inhibition and remarkable resistance to the MEK inhibitor. Additionally, based on proteomics profiling, cell lines in cluster C2 (HEC59, EN, ETN1) were more resistant to the MEK inhibitor cobimetinib $\left(\mathrm{IC}_{50} \mathrm{~s} 3.289,36.511\right.$ and $1.077 \mu \mathrm{M}$, respectively) than cell lines in cluster $\mathrm{C} 3\left(\mathrm{IC}_{50} \mathrm{~s}\right.$ from 0.001 to $\left.0.518 ; P=0.04\right)$, yet again suggesting that PTEN mutations and the level of PTEN protein expression are important events in cell responsiveness to therapies targeted to the PI3K and RAS/ MAPK pathways. Therefore, based on differential sensitivity to the PI3K and MEK inhibitors and differential activation of the PI3K and RAS/MAPK signalling pathways associated with the clustering in Fig. 1b which seems to be largely driven by PTEN, PIK3CA and KRAS mutations and PTEN protein levels, we classified our cell lines into these 4 variables as follows:

Group 1 (PIK3CA mutated EC cell lines): SKUT2, MFE280.

Group 2 (PTEN mutated EC cell lines):

a. (PTEN mutated EC cells with retained PTEN): EN-1078D, MFE296, ETN1.

b. (PTEN mutated EC cells with PTEN Loss): AN3CA, HEC59, EN.

Group 3 (KRAS mutated EC cells): HEC1A, HEC1B, HEC50.

Group 4 (wild-type EC cells): EFE184, KLE (Table 2 and Fig. 2).

In this classification system, PTEN mutations regarded as dominant over PIK3CA mutations because they, and not PIK3CA mutations, are associated with AKT activation as shown earlier. The comparative responsiveness 

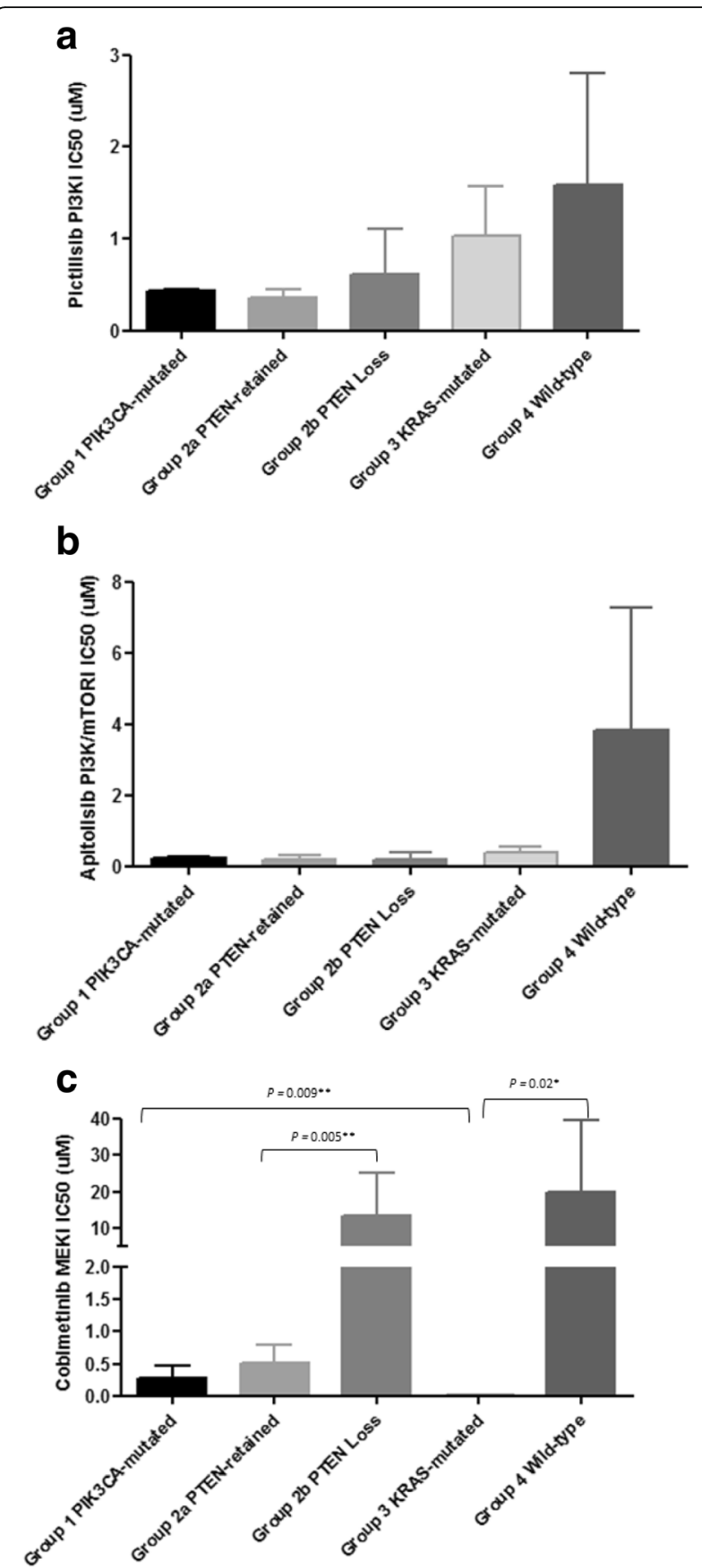

Fig. 2 Comparative inhibitor responsiveness between endometrial cancer cell line groups are shown in Table 2. The figures derived by comparing average $\mathrm{IC}_{50} \mathrm{~s}$ between the groups for each inhibitor alone and in combination a Comparative responsiveness of pictilisib in EC cell line groups, $\mathbf{b}$ Comparative responsiveness of apitolisib in EC cell line groups, c Comparative responsiveness of cobimetinib in EC cell line groups. $P<0.05$ is statistically significant

of each group to the three targeted inhibitors is shown in Fig. 2. Comparison of these groups revealed that group 2a and group $2 \mathrm{~b}$ cell lines with PTEN mutations were most sensitive to the PI3K inhibitor pictilisib, followed by group 1 cell lines with PIK3CA mutations only $\left(\mathrm{IC}_{50}=0.43 \pm 0.01\right)$. In the PTEN-mutated group 2 cell lines, pictilisib responsiveness was independent of PTEN protein expression status. KRAS-mutated cell lines in group 3 and wild-type cell lines in group 4were the most resistant to this inhibitor $\left(\mathrm{IC}_{50}\right.$ values $1.03 \pm$ 0.53 and $1.58 \pm 1.21$, respectively). Group 1 and group 4 cell lines did not differ significantly in their sensitivities to pictilisib, despite the former having and the latter lacking PIK3CA mutations.

Responses to the PI3K/mTOR inhibitor apitolisib overall resembled those to the PI3K inhibitor pictilisib, with for example PTEN-mutated cell lines with retained PTEN (group $2 \mathrm{a}$ ) were being more sensitive (IC50 $=0.17 \pm 0.05$ ) compared to wild-type cell lines in group $4\left(\mathrm{IC}_{50}=0.33 \pm 0.06\right)$ (Fig. 2b).However, group $3 \mathrm{KRAS}$-mutated cell lines were more sensitive to this inhibitor $\left(\mathrm{IC}_{50}=0.19 \pm 0.09 \mu \mathrm{M}\right)$ compared with group 4 cell lines $\left(\mathrm{IC}_{50}=3.81 \pm 3.44 \mu \mathrm{M}\right)$. In general, the differences between the groups in terms of apitolisib sensitivity were not as marked as the differences between the groups in terms of pictilisib sensitivity.

In terms of MEK inhibition, cobimetinib induced a strong inhibition in all except group $2 \mathrm{~b}$ and group $4 \mathrm{EC}$ cell lines $\left(\mathrm{IC}_{50}=10.74 \pm 4.47 \mu \mathrm{M}\right.$ and $1.28 \pm 0.60 \mu \mathrm{M}$, respectively), particularly in group 1 cell lines with PIK3CA mutations $\left(\mathrm{IC}_{50}=0.31 \pm 0.08 \mu \mathrm{M}\right)$ and group 3 KRAS-mutated cell lines $\left(\mathrm{IC}_{50}=0.05 \pm 0.01 \mu \mathrm{M}\right)$ (Fig. $2 \mathrm{c}$ ). However, responses to MEK inhibition varied noticeably between PTEN-mutated cell lines with retained PTEN protein in group $2 \mathrm{a}\left(\mathrm{IC}_{50}=0.31 \pm 0.08 \mu \mathrm{M}\right)$ and PTENmutated cell lines exhibiting loss of PTEN protein in group $2 \mathrm{~b}\left(\mathrm{IC}_{50}=10.74 \pm 4.47 \mu \mathrm{M}\right)$.

Proteomic effects in endometrial cancer cell lines of PI3K inhibitors and a MEK inhibitor

Next, we sought to determine how PI3K and MEK inhibitors affect protein signalling in EC cell lines. We selected a group of $6 \mathrm{EC}$ cell lines, one cell line from the PIK3CA-mutated group 1 (SKUT2), one cell line from the PTEN-mutated PTEN retained cell lines in group 2a (EN-1078D), one cell line from the group $2 b$ cell lines with PTEN loss (AN3CA), two cell lines from the $K R A S$-mutated group 3 (HEC1B; -PIK3CA-mutated, and HEC50; -PIK3CA-wild-type), and finally one cell line from the wild-type group (KLE).

The data in Fig. 4a-e demonstrate changes in phosphorylation and expression of AKT and RAS/MAPK signalling proteins after treatment with $0.1 \mu \mathrm{M}$ of the PI3K, PI3K/ mTOR and MEK inhibitors, and the combinations for $30 \mathrm{~min}$ and $6 \mathrm{~h}$ in $6 \mathrm{EC}$ cell lines. Similar to observation in patients [25], we observed decreases in phosphorylation of AKT following treatment with the PI3K inhibitors pictilisib and apitolisib when compared with control-treated cells. We also observed that reductions in AKT, S6 ribosomal 

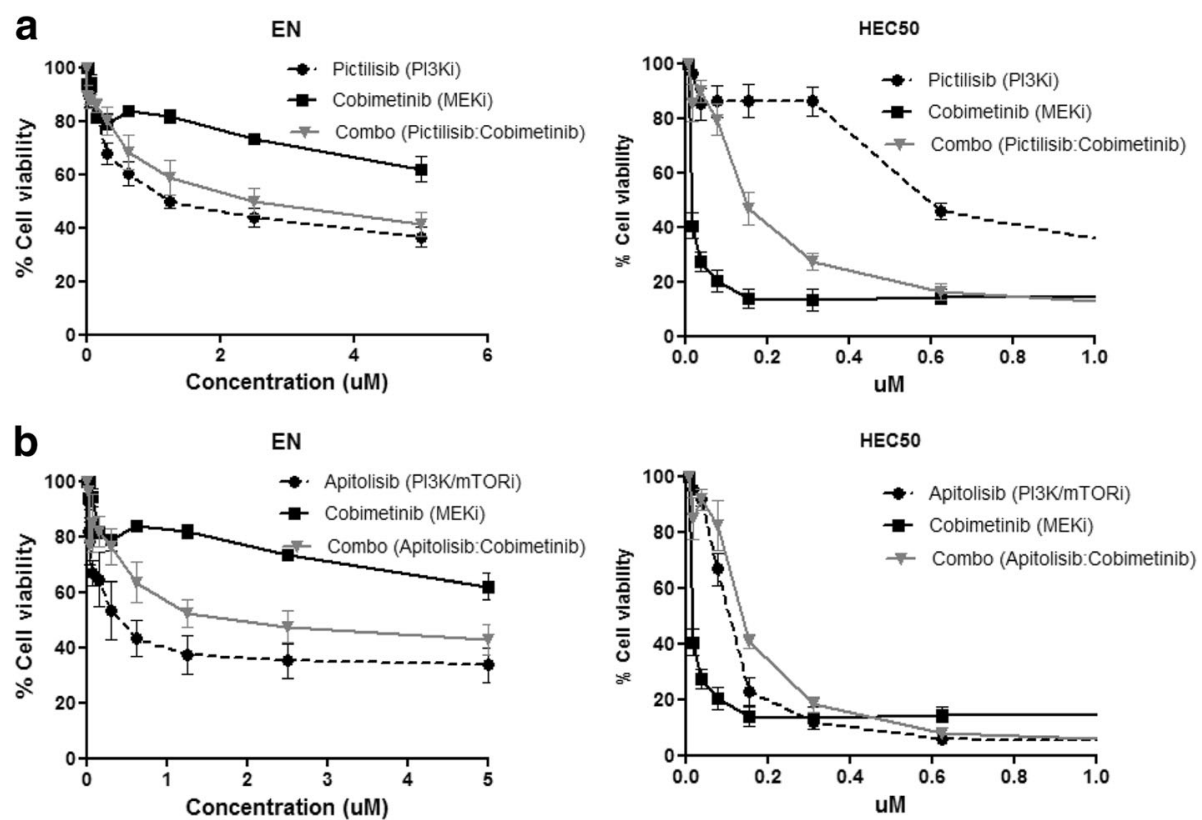

Fig. 3 Endometrial cancer cell lines in which antagonism was observed to the combination of pictilisib (PI3Ki), apitolisib (PI3K/mTORi) and cobimetinib (MEKi). a Two cell lines in which antagonism was observed to the combination of PI3K and MEK inhibition. $\mathbf{b}$ Two cell lines in which antagonism was observed to the combination of PI3K/mTOR and MEK inhibition

protein and GSK-3 $\beta$ phosphorylation occurred in some cell lines. Figure $4 \mathrm{c}$ also demonstrates a general decrease in the phosphorylation of MAPK-ERK1/2 $2^{\text {Thr202/Tyr204 }}$ after treatment with the MEK inhibitor.

We found exposure time to be an important variable, particularly in some EC cells, with some pathways up-regulated immediately after drug exposure down-regulated at longer exposure. For example, $30 \mathrm{~min}$ exposure to each of the PI3K inhibitors and the MEK inhibitor resulted in upregulation of $\mathrm{p} 38 \mathrm{MAPK}^{\mathrm{Thr} 180 / \mathrm{Tyr} 182}$ phosphorylation in the PTEN mutated, PTEN retained cell line EN-1078D, with down-regulation of p38 MAPK $^{\text {Thr180/Tyr182 }}$ phosphorylation at longer exposure (Fig. $4 \mathrm{a}-\mathrm{C}$ ).

While treatment with the PI3K inhibitor pictilisib significantly suppressed the levels of phosphorylated S6 ribosomal protein (especially at Ser ${ }^{240 / 244}$ ), phosphorylated $\mathrm{AKT}$ (at $\mathrm{Thr}^{308}$ and $\mathrm{Ser}^{473}$ ) and phosphorylated GSK-3- $\beta^{\text {Ser9 }}$ in most EC cell lines compared with control-treated cells (Fig. 4a), it significantly induced phosphorylation of MEK $1 / 2^{\text {Ser217/221 }}$, MAPK ${ }^{\text {Thr202/Tyr204 }}$ and $\mathrm{p} 38^{\mathrm{Thr} 180 / \mathrm{Tyr} 182}$ in some cell lines, most notably in the KRAS-mutated cell lines HEC1B and HEC50 at most time point, a likely sign of a feedback loop regulation. While this occurred after $30 \mathrm{~min}$ in $\mathrm{HEC} 1 \mathrm{~B}$, it was more obvious after $6 \mathrm{~h}$ in HEC50, possibly indicating different adaptations by feedback regulation (Fig. 4a). This data suggests that pictilisib-induced activation of the RAS/ MAPK pathway is most likely to occur in $K R A S$-mutated cell lines, possibly underlying the resistance of these cell lines to this PI3K inhibitor.

Consistent with growth inhibition responses to the PI3K/mTOR inhibitor apitolisib resembling those to the PI3K inhibitor pictilisib in the cell line panel, the protein signalling effects of these inhibitors were also very comparable. In general, this inhibitor led to inhibition of AKT phosphorylation in the cell lines in comparison with control-treated cells. As with pictilisib, there was no obvious correlation between degree of the PI3K pathway inhibition and apitolisib sensitivity. Apitolisib induced increases in MEK, MAPK and p38 MAPK phosphorylation were also observed, in particular at $30 \mathrm{~min}$ in the KRAS-mutated cell lines. Of the 6 cell lines tested herein, AN3CA (PTEN-low) and KLE (wild-type) were the most resistant to the MEK inhibitor cobimetinib. While this inhibitor decreased MAPK phosphorylation at $\mathrm{Thr}^{202}$ / $\mathrm{Tyr}^{204}$ in all cell lines (Fig. 4c), its effect seemed to be least marked in these two cell lines. Further, at $6 \mathrm{~h}$, cobimetinib treatment was associated with increased phosphorylation of $A K T^{\text {Ser473 }}$ (in AN3CA) and of GSK- $\beta^{\text {Ser9 }}$ and S6 ribosomal protein ${ }^{\text {Ser240/244 }}$ (in KLE). While MEK phosphorylation at $\operatorname{Ser}^{217 / 221}$ was in general increased in response to cobimetinib, this effect was most marked in the two most sensitive and KRAS-mutated cell lines HEC1B and HEC50 (Fig. 4c). This increase in MEK phosphorylation is likely to be a feedback loop-induced mechanism that seems most marked in the presence of mutated KRAS. 

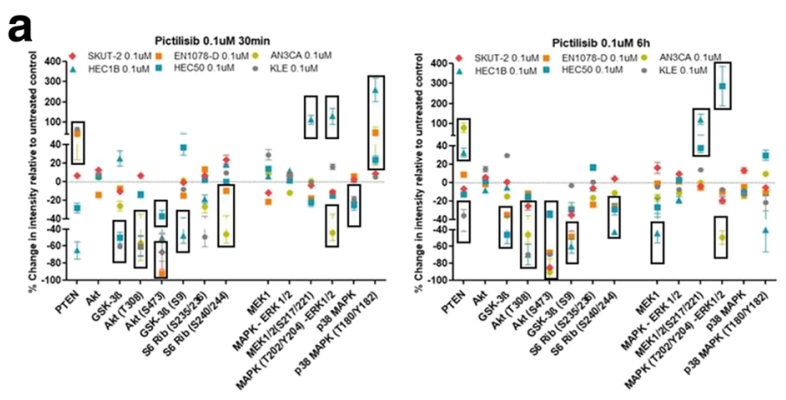

\section{b}

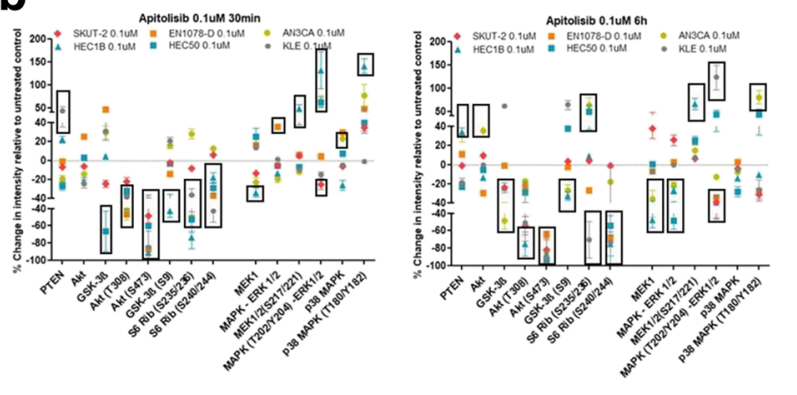

C

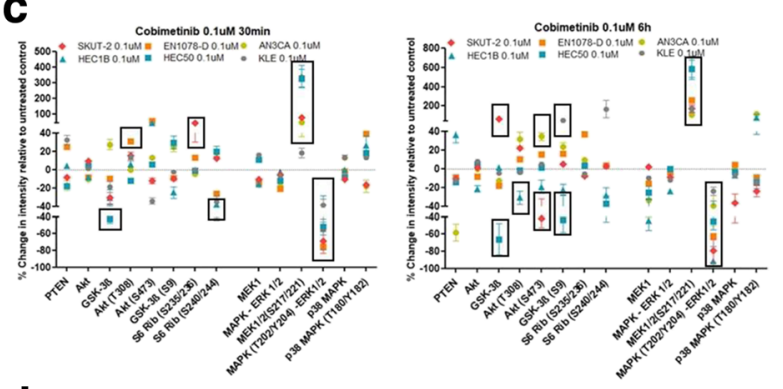

d
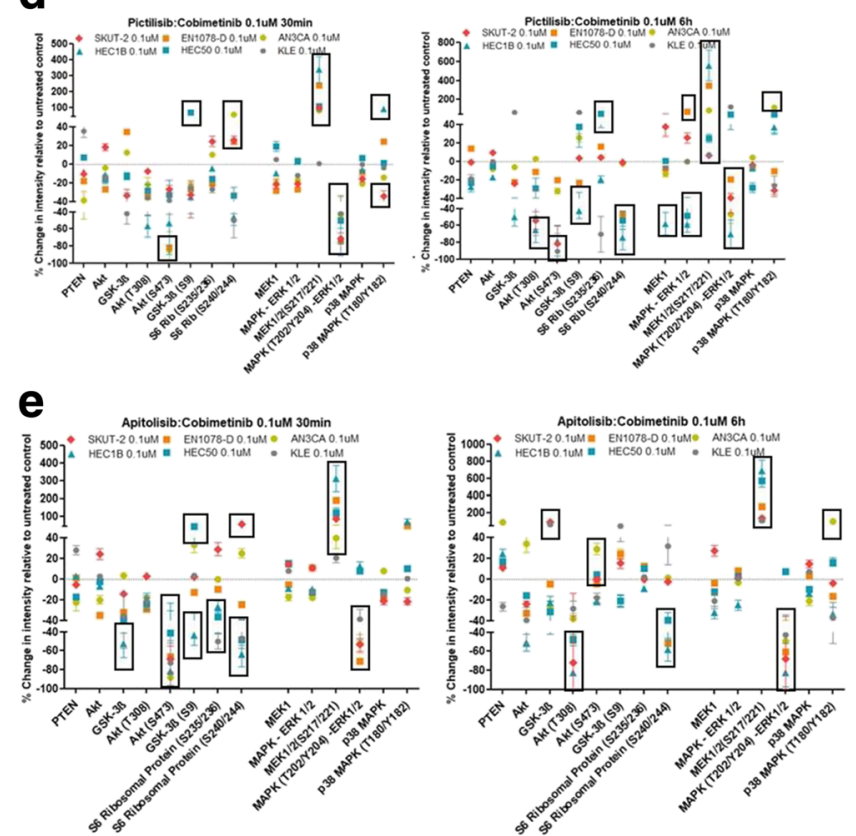

Fig. 4 (See legend on next page.) 
(See figure on previous page.)

Fig. 4 Control-normalized changes in the expression and phosphorylation of PI3K and MAPK signalling proteins induced by $0.1 \mu \mathrm{M}$ concentration at two different time points of the PI3K inhibitors a pictilisib at 30 min and 6 h, b apitolisib at 30 min and 6 h, c cobimetinib at 30 min and 6 h, d the combination of pictilisib with cobimetinib at $30 \mathrm{~min}$ and $6 \mathrm{~h}$, and $\mathbf{e}$ the combination of apitolisib with cobimetinib at $30 \mathrm{~min}$ and $6 \mathrm{~h}$. The specific phosphorylation sites evaluated are indicated (e.g. AKT (T308) in AKT phosphorylation at threonine 308). Cell lines displaying significant differential changes $(P<0.05)$ in protein expression and phosphorylation are shown in boxes

Dual blockage of the PI3K and RAS/MAPK pathways has been shown to synergistically inhibit tumour cell growth in different cancers [11, 26, 27]; including endometrial cancer $[28,29]$. Indeed, we observed that treatment with PI3K and MEK inhibitor combinations was more effective than each inhibitor alone in most EC cell lines tested (9 out of 11) (Table 1). While the PI3K and MEK inhibitor combinations did not abrogate MEK activation induced by cobimetinib alone, they did abrogate the increases in AKT (e.g. in

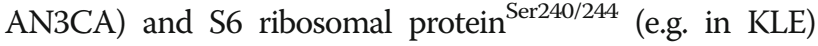
phosphorylation seen with the MEK inhibitor alone (Fig. 4d), possibly contributing to the synergy observed in these cell lines. In HEC50, we also observed that the significant inhibition of MAPK $^{\text {Thr202/Tyr204 }}$ phosphorylation induced by cobimetinib was no longer present with the pictilisib:cobimetinib and apitolisib:cobimetinib combinations, especially at $6 \mathrm{~h}$ (Fig. 4d), possibly contributing to the antagonism seen in this cell line with the drug combinations.

\section{Discussion}

In this study, we profiled the responses of a diverse set of endometrial cancer cell lines to PI3K and MEK inhibitors alone and in combination, to characterise variability in responses by genotype. We found that, in agreement with previous studies [5, 24], mutations in multiple members of the PI3K pathway and KRAS were coexisted in several endometrial cancer cell lines, including concomitant mutations in the PI3K pathway. Several studies have shown that the correlation between mutations in some PI3K pathway members and sensitivity to PI3K inhibitors is weak [30-32].

Classification of tumours based on their genetic signatures and responses to inhibitors is a major goal for advancing targeted personalized therapies. Recent studies proposed to classify endometrial cancers into 4 categories: POLE ultra-mutated, microsatellite instability hypermutated, copy number-low, and copy number-high $[9,33]$. In this study, we focused on the PI3K and RAS/MAPK pathway genomic and proteomic status of EC cell lines and assessed their sensitivity to three selective inhibitors of the PI3K and RAS/MAPK pathways alone and in combination.

We detected differential responsiveness to the PI3K inhibitors and to the MEK inhibitor between the cell lines, with the presence of PTEN mutations and PTEN loss being significantly associated with PI3K pathway activation and enhanced sensitivity to PI3K inhibition and remarkable resistance to the MEK inhibitor. We also examined if proteomic clustering can be used to identify subgroups of cell lines with differential responses to the different targeted therapies.

Indeed, we observed no significant association between PIK3CA or PIK3R1 mutations and AKT phosphorylation, in contrast to PTEN loss and PTEN mutations which were significantly associated with PI3K pathway activation as determined by high levels of AKT phosphorylation. These results are consistent with the previous study by Weigelt et al. [24] and suggest that PTEN mutations and PTEN loss rather than PIK3CA mutations are associated with increased AKT activation in endometrial cancer. Possibly in line with this, we found in our study that PTEN mutations and PTEN loss are dominant predictors of sensitivity to the PI3K inhibitor pictilisib, while PIK3R1, PIK3CA and $K R A S$ are not. Indeed, in our panel of EC cell lines, PIK3CA and KRAS mutations were associated with relative resistance to PI3K inhibitors. While the three KRASmutated cell lines were most sensitive to the MEK inhibitor cobimetinib, PTEN mutations and, in particular PTEN loss, were also dominant biomarkers of resistance to this inhibitor. Our data here suggests a key role for PTEN protein loss in mediating resistance to MEK inhibition, perhaps mediated by a feed-back-loop as suggested previously [13]. This is consistent with the previous study by Hoeflich et al. [13], that also showed loss of PTEN protein to be a negative predictor of response to MEK inhibition.

RPPA analysis revealed that PI3K inhibitors (pictilisib and apitolisib) inhibit AKT phosphorylation in most of the cell lines after $30 \mathrm{~min}$ treatment as expected. However, in KRAS-mutated cell lines, treatment with PI3K inhibitors results in an increase in MEK and MAPK phosphorylation, indicative of a potential link between PI3K inhibition and feedback activation of the RAS/MAPK pathway in these cells, which may contribute to cell line resistance to pictilisib. Thus, PI3K inhibitor-induced RAS/MAPK pathway activation in some cells may explain the ineffectiveness of single agentPI3K inhibition in inhibiting cell growth concurrent targeting of both pathways.

Dual inhibition of the PI3K and RAS/MAPK pathways has been shown to provide a more effective treatment strategy than inhibition of one pathway. In this study, the combinations of the PI3K inhibitor with the MEK inhibitor and of the PI3K/mTOR inhibitor with the MEK inhibitor, showed additivity or synergy in inhibiting the growth of most of the cell lines tested, with the exception of two cell lines. One of these cell lines had a KRAS mutation only without a PI3K 
pathway mutation (HEC50), and the antagonism of the combinations may possibly be partly as a result of the particularly marked anti-tumour efficacy of MEK inhibition alone in this cell line. Our RPPA data showed that treatment of HEC50 cells with the MEK inhibitor alone inhibited MAPK phosphorylation more effectively than the combination of the PI3K and MEK inhibitors, possibly contributing to this antagonism. Indeed, with the combination treatments, MAPK phosphorylation at $\mathrm{Thr}^{202} / \mathrm{Tyr}^{204}$ was not significantly inhibited in HEC50, in contrast to the effect of cobimetinib alone. This data suggests that dual blockade of PI3K with MEK may not improve the efficacy of MEK inhibition in some KRAS-mutated EC cell lines that do not possess a PI3K pathway mutation.

In terms of developing a classification system that integrates PI3K and RAS/MAPK genomic events to predict the likelihood of inhibitor response, the dominance of PTEN mutations and PTEN protein loss as predictors of AKT activation and drug response, make it reasonable to center classification surrounding these events. As KRAS mutations are also associated with resistance to PI3K inhibition and other targeted therapies (e.g. EGFR), we felt it is reasonable to define another cell line subgroup based on these mutations. This left four cell lines, two with PIK3CA mutations only, and two with no mutations in PIK3CA, KRAS, PTEN, PIK3R1or PIK3R2, which were subdivided into PIK3CA mutation alone and wild-type subgroups. Overall, this classification system, shown in Table 2, offers the potential to classify endometrial cancers based on responsiveness to PI3K and MEK inhibitors.

\section{Conclusion}

In summary, inhibition of MEK with cobimetinib alone or in combination with PI3K inhibitors (pictilisib and apitolisib) could be a promising treatment modality, not just for endometrial cancer patients with mutated KRAS, but also for those with retained PTEN expression. Up-regulation of MEK/MAPK signalling by PI3K inhibition, and up-regulation of AKT activation by MEK inhibition may serve as potential biomarkers of likely responsiveness to each inhibitor. Combinations of PI3K and MEK inhibitors are potentially promising for the treatment of many ECs.

\section{Additional files}

Additional file 1: Table S1. The 66 primary antibodies used in RPPA in this study are listed. The phosphorylation sites (amino acids) for phospho-specific antibodies are shown. (XLSX $10 \mathrm{~kb}$ )

Additional file 2: Table S2. Baseline levels of 13 EC cell lines for the 66 primary antibodies used in RPPA are listed and t-test result for the comparison between PTEN-mutated EC and wild-type-PTEN EC cell lines shown. Significant associations are in highlighted in pink. (XLSX 21 kb)

Additional file 3: Figure S1. Endometrial cancer cell lines in which synergism was observed to the combination of pictilisib (PI3K), apitolisib (PI3K/mTOR), and cobimetinib (MEK) inhibitors. a) Eight cell lines in which synergism was observed to the combination of PI3K and MEK inhibition. b) Eight cell lines in which synergism was observed to the combination of PI3K/mTOR and MEK inhibition. (XLSX $158 \mathrm{~kb}$ )

\section{Abbreviations}

AKT: Also known as protein kinase B, serine/threonine-specific protein kinase; Cl: Combination index; DMEM: Dulbecco's modified eagle medium;

DMSO: Dimethyl sulfoxide; DNA: Deoxyribonucleic acid; EC: Endometrial cancer; ED75: Effective dose exhibiting $75 \%$ of loss in cell viability; FBS: Fetal bovine serum; HEPES: 4-(2-hydroxyethyl)-1-piperazineethanesulfonic acid; IC50: Half maximal inhibitory concentration; KRAS : Kirsten ras oncogene homolog; MEK: Also known as MAPK, Mitogen-activated protein kinase; MEKi: MEK inhibitor; MEM: Minimum essential medium; mTOR: Mechanistic target of rapamycin; PBS: Phosphate buffered saline; PI3K: Phosphoinositide3-kinase; PI3K/mTORi: PI3K/mTOR inhibitor; PI3Ki: PI3K inhibitor;

PIK3CA: Phosphatidylinositol-4,5-bisphosphate 3-kinase catalytic subunit alpha; PIK3R1: Phosphoinositide-3-kinase regulatory subunit 1:

PIK3R2: Phosphoinositide-3-kinase regulatory subunit 2; PTEN: Phosphatase and tensin homolog; RAS/MAPK: Mitogen-activated protein kinase pathway; RPMI: Roswell Park Memorial Institute medium; RPPA: Reverse phase protein array; SEM: Standard error of mean

\section{Acknowledgements}

We thank Dr. Klaus Hoeflich (Genentech USA, Inc.) for providing us with pictilisib, apitolisib and cobimetinib inhibitors and Prof Eric Asselin (University of Quebec) for providing us with EN-1078D cell line. We also thank Dr. Katherine Stemke-Hale (the Characterized Cell Line Core facility at MDACC, the University of Texas) for help with the somatic mutation genotyping (MassARRAY, Sequenom) analyses.

\section{Funding}

This research was funded by the Irish Cancer Society's first Collaborative Cancer Research Centre BREAST-PREDICT (grant CCRC13GAL), the National Cancer Institute (grants P30 CA016672 and P50 CA098258), the North East Cancer Research and Education Trust, and the Health Research Board/Science Foundation Ireland (HRB-SFI) Translational Research Award (grant TRA 2010/08). The funding body had no role in the design of the study and collection, analysis, and interpretation of data and in writing the manuscript.

\section{Availability of data and materials}

All data supporting the findings in this study are included within the manuscript and its Additional files 1,2 and 3. The datasets used and/or analysed during the current study are available from the corresponding author on reasonable request.

\section{Authors' contributions}

$\mathrm{OA}$ and $\mathrm{BTH}$ designed and completed this study and wrote the manuscript. $M C$ and CM assisted with the experiments concerning analysis of RPPA. LWC and GBM helped with the mutational analysis and contributed intellectually to the study. All authors have read and approved the final manuscript.

\section{Ethics approval and consent to participate}

All endometrial cancer cell lines used in this study are commercially available with the exception of E1078D cell line which was kindly provided by Prof Eric Asselin (University of Quebec). The informed consent for EN1078D cell line was attained and research studies approved by the Montreal University Institutional Review Board.

\section{Consent for publication}

Not applicable.

\section{Competing interests}

The authors declare that they have no competing interests.

\section{Publisher's Note}

Springer Nature remains neutral with regard to jurisdictional claims in published maps and institutional affiliations. 


\section{Author details}

'Department of Medical Oncology, Royal College of Surgeons in Ireland, Beaumont Hospital, Dublin 9, Ireland. ${ }^{2}$ Department of Systems Biology, the University of Texas M.D. Anderson Cancer Center, Houston, TX 77030, USA.

Received: 19 July 2016 Accepted: 23 January 2018

Published online: 09 February 2018

\section{References}

1. Oda K, et al. High frequency of coexistent mutations of PIK3CA and PTEN genes in endometrial carcinoma. Cancer Res. 2005;65(23):10669-73.

2. Hennessy BT, et al. Exploiting the PI3KJAKT pathway for cancer drug discovery. Nat Rev Drug Discov. 2005;4(12):988-1004.

3. Gupta S, et al. Binding of ras to phosphoinositide 3-kinase p110alpha is required for ras-driven tumorigenesis in mice. Cell. 2007:129(5):957-68.

4. Urick ME, et al. PIK3R1 (p85alpha) is somatically mutated at high frequency in primary endometrial cancer. Cancer Res. 2011;71(12):4061-7.

5. Cheung LW, et al. High frequency of PIK3R1 and PIK3R2 mutations in endometrial cancer elucidates a novel mechanism for regulation of PTEN protein stability. Cancer Discov. 2011;1(2):170-85.

6. Velasco A, et al. PIK3CA gene mutations in endometrial carcinoma: correlation with PTEN and K-RAS alterations. Hum Pathol. 2006:37(11):1465-72.

7. Kang $\mathrm{S}$, et al. Mutual exclusiveness between PIK3CA and KRAS mutations in endometrial carcinoma. Int J Gynecol Cancer. 2008;18(6):1339-43.

8. Folkes AJ, et al. The identification of 2-(1H-indazol-4-yl)-6-(4-methanesulfonylpiperazin-1-ylmethyl)-4-morpholin-4-yl-t hieno[3,2-d]pyrimidine (GDC-0941) as a potent, selective, orally bioavailable inhibitor of class I PI3 kinase for the treatment of cancer. J Med Chem. 2008;51(18):5522-32.

9. Talhouk A, et al. A clinically applicable molecular-based classification for endometrial cancers. Br J Cancer. 2015;113(2):299-310.

10. Wallin JJ, et al. GDC-0980 is a novel class I PI3K/mTOR kinase inhibitor with robust activity in cancer models driven by the PI3K pathway. Mol Cancer Ther. 2011;10(12):2426-36.

11. Hoeflich KP, et al. Intermittent administration of MEK inhibitor GDC-0973 plus PI3K inhibitor GDC-0941 triggers robust apoptosis and tumor growth inhibition. Cancer Res. 2012;72(1):210-9.

12. Xie $\mathrm{C}$, et al. Mechanisms of synergistic antileukemic interactions between valproic acid and cytarabine in pediatric acute myeloid leukemia. Clin Cancer Res. 2010;16(22):5499-510.

13. Hoeflich KP, et al. In vivo antitumor activity of MEK and phosphatidylinositol 3-kinase inhibitors in basal-like breast cancer models. Clin Cancer Res. 2009; 15(14):4649-64.

14. Dery MC, et al. Characterization of EN-1078D, a poorly differentiated human endometrial carcinoma cell line: a novel tool to study endometrial invasion in vitro. Reprod Biol Endocrinol. 2007:5:38.

15. Stemke-Hale $K$, et al. An integrative genomic and proteomic analysis of PIK3CA PTEN, and AKT mutations in breast cancer. Cancer Res. 2008;68(15):6084-91.

16. Martin A, Clynes M. Comparison of 5 microplate colorimetric assays for in vitro cytotoxicity testing and cell proliferation assays. Cytotechnology. 1993; 11(1):49-58

17. Stordal $B$, et al. BRCA1/2 mutation analysis in 41 ovarian cell lines reveals only one functionally deleterious BRCA1 mutation. Mol Oncol. 2013;7(3):567-79.

18. Wiegand $\mathrm{KC}$, et al. A functional proteogenomic analysis of endometrioid and clear cell carcinomas using reverse phase protein array and mutation analysis: protein expression is histotype-specific and loss of ARID1A BAF250a is associated with AKT phosphorylation. BMC Cancer. 2014;14:120.

19. Tibes $R$, et al. Reverse phase protein array: validation of a novel proteomic technology and utility for analysis of primary leukemia specimens and hematopoietic stem cells. Mol Cancer Ther. 2006:5(10):2512-21.

20. Zhang $L$, et al. Serial dilution curve: a new method for analysis of reverse phase protein array data. Bioinformatics. 2009:25(5):650-4.

21. Hennessy BT, et al. Pharmacodynamic markers of perifosine efficacy. Clin Cancer Res. 2007;13(24):7421-31.

22. Chou TC, Talalay P. Quantitative analysis of dose-effect relationships: the combined effects of multiple drugs or enzyme inhibitors. Adv Enzym Regul. 1984:22:27-55

23. Liang $\mathrm{H}$, et al. Whole-exome sequencing combined with functional genomics reveals novel candidate driver cancer genes in endometrial cancer. Genome Res. 2012;22(11):2120-9.

24. Weigelt B, et al. PI3K pathway dependencies in Endometrioid endometrial cancer cell lines. Clin Cancer Res. 2013;19(13):3533-44.
25. Hoang LN, et al. Histotype-genotype correlation in 36 high-grade endometrial carcinomas. Am J Surg Pathol. 2013;37(9):1421-32.

26. Renshaw J, et al. Dual blockade of the PI3K/AKT/mTOR (AZD8055) and RAS/ MEK/ERK (AZD6244) pathways synergistically inhibits rhabdomyosarcoma cell growth in vitro and in vivo. Clin Cancer Res. 2013;19(21):5940-51.

27. Qu Y, et al. Antitumor activity of selective MEK1/2 inhibitor AZD6244 in combination with PI3K/mTOR inhibitor BEZ235 in gefitinib-resistant NSCLC xenograft models. J Exp Clin Cancer Res. 2014;33:52.

28. Inaba $\mathrm{K}$, et al. Antitumor activity of a combination of dual PI3K/mTOR inhibitor SAR245409 and selective MEK1/2 inhibitor pimasertib in endometrial carcinomas. Gynecol Oncol. 2015:138(2):323-31.

29. Schrauwen $\mathrm{S}$, et al. Dual blockade of PI3K/AKT/mTOR (NVP-BEZ235) and Ras/Raf/MEK (AZD6244) pathways synergistically inhibit growth of primary endometrioid endometrial carcinoma cultures, whereas NVPBEZ235 reduces tumor growth in the corresponding xenograft models. Gynecol Oncol. 2015;138(1):165-73.

30. Janku F, et al. PI3K/AKT/mTOR inhibitors in patients with breast and gynecologic malignancies harboring PIK3CA mutations. J Clin Oncol. 2012;30(8):777-82.

31. Dan S, et al. Correlating phosphatidylinositol 3-kinase inhibitor efficacy with signaling pathway status: in silico and biological evaluations. Cancer Res. 2010;70(12):4982-94

32. Casado P, et al. Phosphoproteomics data classify hematological cancer cell lines according to tumor type and sensitivity to kinase inhibitors. Genome Biol. 2013;14(4):R37.

33. Cancer Genome Atlas Research, N, et al. Integrated genomic characterization of endometrial carcinoma. Nature. 2013;497(7447):67-73.

\section{Submit your next manuscript to BioMed Central and we will help you at every step:}

- We accept pre-submission inquiries

- Our selector tool helps you to find the most relevant journal

- We provide round the clock customer support

- Convenient online submission

- Thorough peer review

- Inclusion in PubMed and all major indexing services

- Maximum visibility for your research

Submit your manuscript at www.biomedcentral.com/submit
) Biomed Central 